\title{
Delayed recurrent spontaneous pneumothorax post-recovery from COVID-19 infection
}

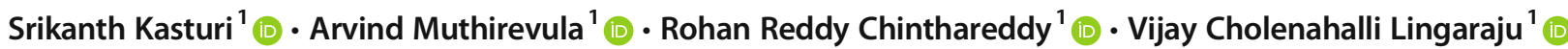

Received: 23 October 2020 / Revised: 30 December 2020 / Accepted: 6 January 2021 / Published online: 2 February 2021

(C) Indian Association of Cardiovascular-Thoracic Surgeons 2021

\begin{abstract}
Pneumothorax, as a consequence of coronavirus disease 2019 (COVID-19) infection, has become an established entity but the delayed occurrence of pneumothorax, after recovery from the illness, is less commonly reported. We present a case of delayed recurrent spontaneous pneumothorax, presenting 4 weeks after recovery from COVID-19 in a previously healthy middle-aged gentleman, for which uniportal video-assisted thoracoscopic surgery (VATS) pleurectomy was performed, but the cause of pneumothorax could not be ascertained. This report brings to light, the importance of continued surveillance of COVID-19 survivors, the unpredictability of the disease process, and the challenges of thoracic surgery in this unique subset of patients.
\end{abstract}

Keywords COVID-19 $\cdot$ Delayed recurrent pneumothorax $\cdot$ Uniportal VATS $\cdot$ Pleurodesis $\cdot$ Pleurectomy

\section{Introduction}

Since the first report of a cluster of cases from Wuhan, China, the coronavirus disease 2019 (COVID-19) caused by the severe acute respiratory syndrome coronavirus-2 (SARS-CoV2) has grown out of proportion, baffling the healthcare systems worldwide. The long-term complications of the disease are presently unknown. It was initially thought to cause pulmonary parenchymal damage, but the extra-parenchymal manifestations eventually became evident in the form of pulmonary thromboembolism, pleural effusion, empyema, and pneumothorax, to name a few. We present a case of delayed recurrent multiloculated pneumothorax as a sequel to COVID19 infection and its management with uniportal video-assisted thoracoscopic surgery (VATS). Informed patient consent and ethics committee approval were obtained.

Vijay Cholenahalli Lingaraju

drvijaycl@yahoo.com

1 Department of Thoracic Surgery, Mazumdar Shaw Medical Center, 258/A, Hosur Road, Bommasandra Industrial Area,

Bengaluru, Karnataka 560099, India

\section{Case report}

A 55-year-old gentleman, never-smoker, presented to us with low-grade fever and he tested positive for COVID-19. He was intubated on day 3 for hypoxia, required mechanical ventilation for 10 days (Fig. 1a), and he was discharged after 35 days of admission. He did not have any evidence of secondary bacterial infection. Four weeks later, he presented with sudden onset dyspnea and right-sided pleuritic chest pain, with a room air saturation of $84 \%$. Computed tomography (CT) of the chest showed a multiloculated right-sided pneumothorax (Fig. 1b, c), for which an intercostal drain (ICD) was inserted. Chest imaging showed a partial resolution; hence, an apical ICD was subsequently inserted. Forced expiratory air leak was present in both the ICDs, which settled after 3 days. After clinical and radiological resolution of pneumothorax, the ICDs were removed and the patient was discharged 2 weeks later. However, he had a similar presentation 2 days later and an ICD was re-inserted. Following a $\mathrm{CT}$ chest showing incomplete lung expansion, despite an ICD in situ (Fig. 1d, e) with negative suction, he was planned for diagnostic thoracoscopy. Uniportal VATS approach was chosen. The lung was adherent at multiple sites, creating loculi that were lysed (Fig. 2). No blebs, bullae, or an active source of air leak was noted. A partial parietal pleurectomy with mechanical abrasion of the lower costal and diaphragmatic pleura was performed, as a form of pleurodesis. Blood loss was not significant $(<150 \mathrm{ml})$. 


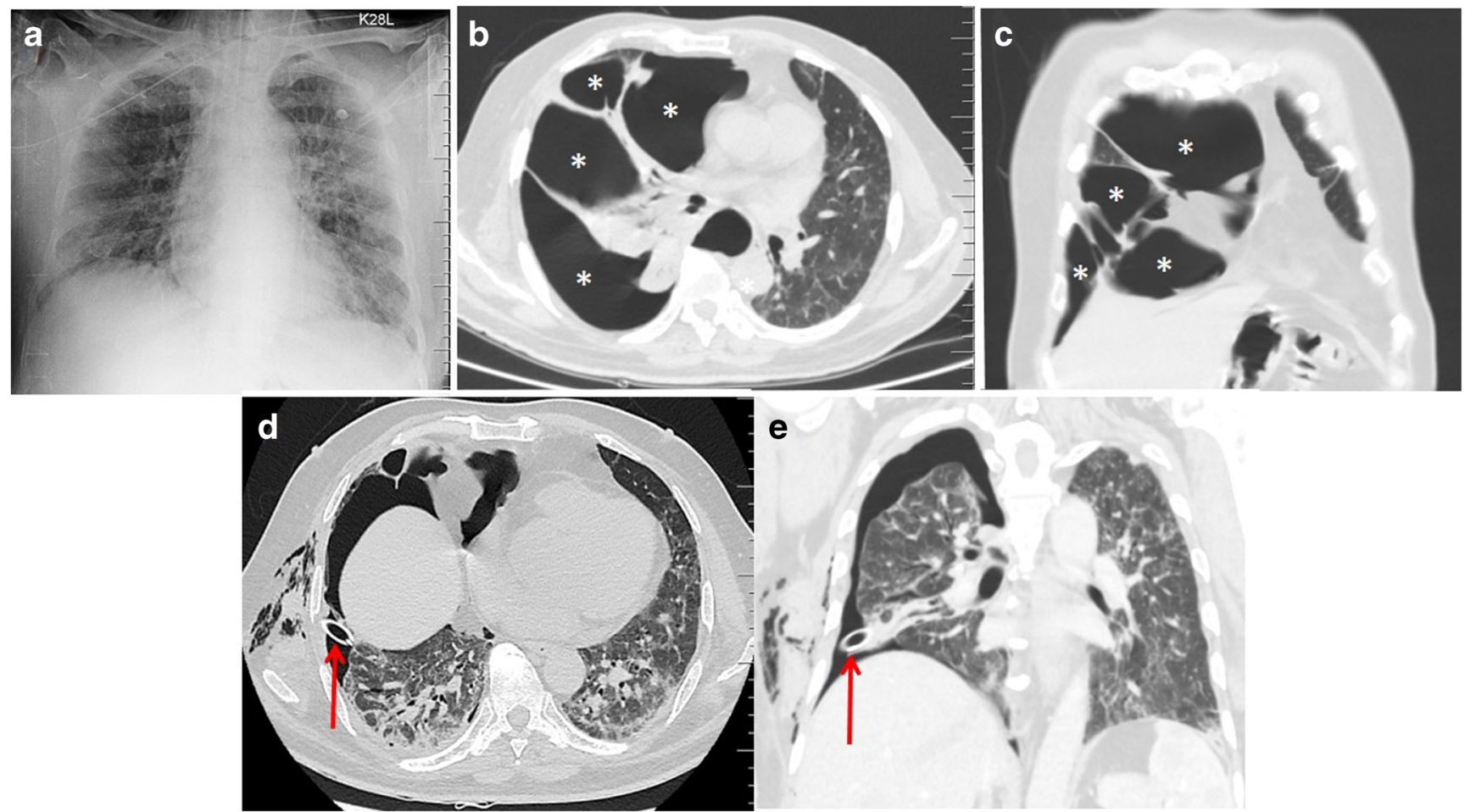

Fig. 1 a Chest X-ray during initial admission with COVID-19 showing bilateral reticular infiltrates consistent with ARDS requiring mechanical ventilation. CT scan done during readmission, 4 weeks later, showing multiloculated right pneumothorax (asterisks) with compression atelectasis of the right upper and middle lobes in axial (b) and coronal (c) sections

The ICD was removed on postoperative day 3, after achieving complete lung expansion (Fig. 3) and the patient was discharged.

\section{Discussion}

Spontaneous pneumothorax as a complication of COVID-19 infection has been described, with a majority implicating barotrauma, following mechanical ventilation in an already compromised lung, as a probable etiology [1,2]. The uniqueness of our case lies in its delayed presentation and recurrence, with incomplete lung expansion despite drainage, necessitating

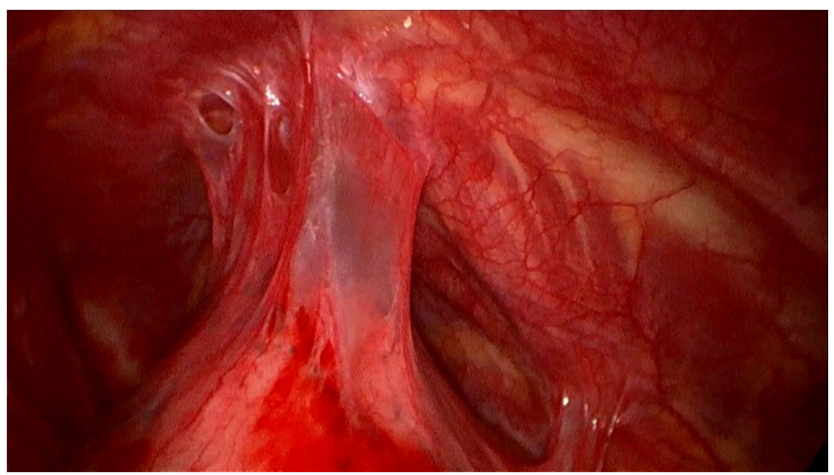

Fig. 2 Intra-operative thoracoscopic image showing lung adhesions creating loculi with mediastinal shift to the left. CT scan done after ICD insertion during readmission for recurrence shows an unexpanded right lung with an ICD in situ (red arrows) in axial (d) and coronal (e) sections (COVID-19, coronavirus disease 2019; ARDS, acute respiratory distress syndrome; $\mathrm{CT}$, computed tomography; ICD, intercostal drain)

surgical intervention, and the absence of bullae or blebs on thoracoscopy. The possible mechanism of COVID-related pneumothorax is attributed to pulmonary parenchymal injury, alveolar membrane damage, and bulla formation [3], which when subjected to high intra-alveolar pressure during acts such as coughing or high positive end-expiratory pressure ventilation results in pneumothorax. Our patient presented to us with pneumothorax, almost 50 days after being off positive pressure ventilation. We hypothesize that the delayed

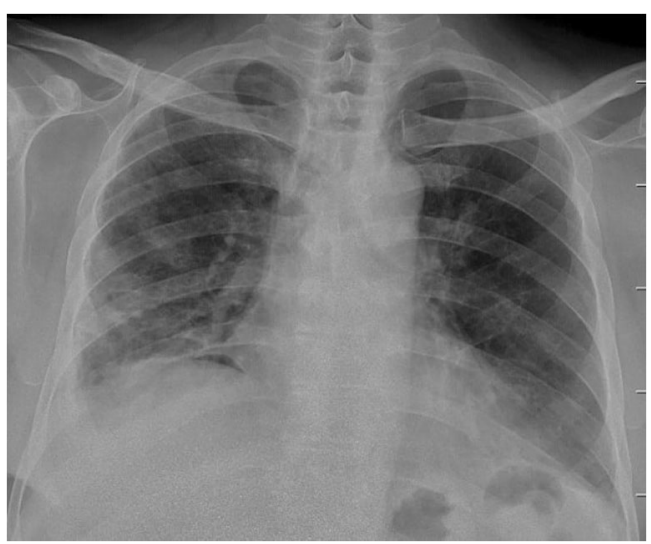

Fig. 3 Chest X-ray after uniportal VATS pleurectomy/pleurodesis showing no residual pneumothorax (VATS, video-assisted thoracoscopic surgery) 
presentation of such pneumothoraces is due to the persistent chronic inflammatory changes and a delayed alveolar breach as part of an ongoing chronic disease process, which is yet to be fully understood [4]. Ruptured small subpleural blebs inciting the primary episode of pneumothorax, but later sealing off spontaneously, only to reopen later, could explain the recurrence. The multiple loculations can be ascribed to a previous inflammatory reaction, secondary to COVID-related acute respiratory distress syndrome, which could cause pleural adhesions creating septa within the pleural space.

Earlier reports described delayed pneumothoraces, which were managed with intercostal drainage only $[5,6]$. From our experience, we agree with Aiolfi et al. [7] that early intervention, through a minimally invasive approach, would offer better outcomes in these patients to prevent the recurrence of pneumothorax and its associated complications. The challenges of operative management of recurrent pneumothorax, in patients who have recovered from COVID-19, are manifold: exposure of healthcare workers to aerosol-generating procedures (AGPs), maintenance of one-lung ventilation, the hazard of developing contralateral pneumothorax, and the dubious quality of the underlying lung tissue to withstand resection. Though the reverse transcriptase-polymerase chain reaction (RT-PCR) for SARS-CoV-2 was negative in our patient prior to surgery, all associated healthcare workers donned level III personnel protective equipment (PPE), given the uncertainty regarding the sensitivity of PCR testing, risk of prolonged viral shedding, and the chance of re-infection. The role of PPE in this clinical setting is debatable, with emerging evidence showing low infectivity risk in immunocompetent individuals. However, individual institutional protocols should guide the operating team. We could successfully maintain one-lung ventilation, probably due to the recovery of the contralateral lung following a delay in presentation. As no bulla or air leak was noted in our patient, we decided to do pleurodesis to prevent recurrent pneumothorax. Talc was not used as it is known to cause respiratory distress in some patients [8]. Gine et al. [9], in a recent report, expressed concerns of coagulopathy and bleeding in COVID-19 patients precluding surgical pleurodesis. No significant bleeding was noted in our case, which can be partly attributable to the late presentation.

\section{Conclusion}

With skyrocketing numbers of COVID-19 infections worldwide, knowledge of the long-term sequelae of this illness will enable us to tackle them expeditiously. This case emphasizes the importance of long-term follow-up and close surveillance of COVID-19 survivors, to understand the natural history of the disease and to offer timely and appropriate care. Thoracic surgery can be safely conducted in this group of patients, with certain precautions to protect the healthcare personnel, with minimally invasive options being better suited to this already debilitated patient population.

Author contributions All authors contributed to the study conception. The first draft of the manuscript was written by Srikanth Kasturi and all authors commented on previous versions of the manuscript. All authors read and approved the final manuscript.

\section{Funding None.}

\section{Compliance with ethical standards}

Ethics approval The Narayana Health Academic Ethics Committee (NHAEC) approved this case report for publication (letter no. NHH/ AEC-CL-2020-578 dated 15.09.2020).

Consent for publication Informed patient consent for publication of this case report was obtained.

Conflict of interest The authors have no conflicts of interest to declare that are relevant to the content of this article.

\section{References}

1. Chen N, Zhou M, Dong X, et al. Epidemiological and clinical characteristics of 99 cases of 2019 novel coronavirus pneumonia in Wuhan, China: a descriptive study. Lancet. 2020;395:507-13.

2. Martinelli AW, Ingle T, Newman J, et al. COVID-19 and pneumothorax: a multicentre retrospective case series. Eur Respir J. 2020;56: 2002697.

3. Yasukawa K, Vamadevan A, Rollins R. Bulla formation and tension pneumothorax in a patient with COVID-19. Am J Trop Med Hyg. 2020;103:943-4.

4. Borczuk AC, Salvatore SP, Seshan SV, et al. COVID-19 pulmonary pathology: a multi-institutional autopsy cohort from Italy and New York City. Mod Pathol. 2020;33:2156-68. https://doi.org/10.1038/ s41379-020-00661-1.

5. Hollingshead C, Hanrahan J. Spontaneous pneumothorax following COVID-19 pneumonia. ID Cases. 2020;21:e00868.

6. Shah VM, Brill KL, Dhingra G, Kannan SG. Delayed recurrent spontaneous pneumothorax in a patient recovering from COVID19 pneumonia. Korean J Anesthesiol. 2020. https://doi.org/10. 4097/kja.20400.

7. Aiolfi A, Biraghi T, Montisci A, et al. Management of persistent pneumothorax with thoracoscopy and blebs resection in COVID19 patients. Ann Thorac Surg. 2020;110:e413-5. https://doi.org/10. 1016/j.athoracsur.2020.04.011.

8. de Campos JR, Vargas FS, de Campos WE, et al. Thoracoscopy talc poudrage : a 15-year experience. Chest. 2001;119:801-6.

9. Giné C, Laín A, García L, López M. Thoracoscopic bullectomy for persistent air leak in a 14-year-old child with COVID-19 bilateral pulmonary disease. J Laparoendosc Adv Surg Tech A. 2020;30:9358.

Publisher's note Springer Nature remains neutral with regard to jurisdictional claims in published maps and institutional affiliations. 